\title{
In vitro and in vivo Study of a Novel Liposome- Mediated Dual Drug Delivery for Synergistic Lung Cancer Therapy via Oral Administration
}

This article was published in the following Dove Press journal: OncoTargets and Therapy

\section{Qi Zhou}

Zhiqiang $\mathrm{Fu}$

Department of Thoracic Surgery, Shanghai Shidong Hospital, Yangpu District, Shanghai 200438, People's Republic of China
Correspondence: Zhiqiang Fu Tel +86 2I-25066666

Email Fuzhiqiangl31I@163.com
Background: To establish the co-delivery liposomes of gefitinib (GFT) and curcumin (CUR) via oral administration with the goals of improving the synergistic effect and reducing acquired drug resistance.

Methods: We prepared liposomes (LPs) which can embed the anticancer compound GFT and CUR and investigated whether they could enhance the antitumor effects of anticancer drugs against MDR. The LPs system was characterized by transmission electron microscopy (TEM), particle size, encapsulation efficiency, cellular uptake and cell viability. In addition, the release characteristics and pharmacodynamics of the LPs were also studied in detail.

Results: The results showed that GFT/CUR LPs were characterized by small particle size of about $130 \mathrm{~nm}$ and negative zeta potential of about $-22.2 \mathrm{mV}$, and the drug controlled to release slowly on a biphasic pattern. Compared with control groups, GFT/CUR LPs showed a higher cellular uptake and cell inhibition rates. Through pharmacodynamics analysis, we found that two compounds (GFT and CUR) were incorporated into one LPs carrier, which played a good role in synergistic effect.

Conclusion: Co-delivery of GFT and CUR has the potential to improve cancer treatment efficacy and overcome acquired resistance, especially towards GFT-resistant cells.

Keywords: gefitinib, curcumin, co-delivery, cellular uptake, cell viability, pharmacodynamics

\section{Introduction}

Non-small cell lung cancer (NSCLC) causes $14 \%$ of all cancer-related deaths and is by far one of the most deadly cancers for both men and women. The survival rate of NSCLC patients varies depending on the extent (stage) of cancer. The 5 -year survival rate of patients with locally advanced non-small cell lung cancer (stage III or above) is $5 \%$ to $36 \%$, and the estimated survival rate varies through different stages of diagnosis. ${ }^{1}$ Active treatments of NSCLC include surgery, radiotherapy and chemotherapy, which can be applied in the form of single therapy or combined therapy. Significant advances have been made in medical treatment in recent years; however, they are not enough to substantially affect the high mortality and morbidity associated with lung cancer. Recently, two oncogenic driving factors, epidermal growth factor receptor (EGFR) mutation and ALK fusion, have been identified. Tyrosine kinase inhibitors targeting EGFR have succeeded in the treatment of NSCLC. Using EGFR inhibitors to treat cancer patients with EGFR mutations can effectively alleviate pain, inhibit tumor metastasis, and even kill cancer cells. ${ }^{2-4}$ 
Gefitinib (GFT), as the first selective inhibitor of EGFR tyrosine kinase domain, is widely used in the chemical therapy of many kinds of tumors. ${ }^{5}$ It belongs to Biopharmaceutical Classification System class II and acts by inhibiting EGFR tyrosine kinase. Its low solubility (sparingly soluble at $\mathrm{pH} 1$ ) in upper gastric fluid affects the onset of action, bioavailability, and therapeutic activity. The logarithm $P$ value of GFT is 4.15 , which indicates that GFT has strong hydrophobicity. The daily oral dose is $250 \mathrm{mg}$ and the bioavailability is $44 \%{ }^{6}$ The most common adverse drug reactions are hepatobiliary diseases, gastrointestinal diseases, metabolism and nutrition disorders, skin and subcutaneous diseases, etc. ${ }^{7}$ Therefore, it is necessary to improve the oral bioavailability of GFT and reduce the daily oral dose. However, unfortunately, after a period of drug exposure, some patients' response to GFT will be greatly reduced due to an effect called acquired resistance. ${ }^{8-11}$

Curcumin (CUR) is a polyphenol extracted from the rhizome of Curcuma longa Linn (turmeric). ${ }^{12,13}$ It exhibits a variety of pharmacological activities, including antioxidant, anti-inflammatory, antibacterial and antitumor effects. ${ }^{14-16}$ Furthermore, some studies have shown that CUR independently exhibits P-gp inhibitory activity by down-regulation of the PI3K/Akt and NF-kB pathways. ${ }^{17,18}$ Therefore, CUR could be used as an effective P-gp inhibitor for multi-drug resistance (MDR)-expressing cancer cells by coadministration with an anticancer drug to maximize the cytotoxicity of the anticancer drug. ${ }^{19}$

In this study, DSPE-PEG 2000 LPs entrapping both anticancer drug GFT and CUR were prepared and their ability to enhance the delivery of anticancer drugs against tumoracquired resistance was determined. The LPs system was characterized by transmission electron microscopy (TEM), particle size and encapsulation efficiency. In addition, the release characteristics and pharmacodynamics of the LPs were also studied in detail. Although both compounds have been reported to be used in lung cancer, few of them are combined in the one drug delivery system. Through this research, we hope to use a carrier platform that simultaneous delivery of GFT and CUR by LPs might be a promising treatment to reverse acquired resistance in tumor cells.

\section{Materials and Methods}

\section{Materials}

GFT was purchased from DiBo Chemical Co., Ltd (Hubei, China) and CUR, from Jianhe Biopharm Co., Ltd
(Shaanxi, China). 1,2-Distearoyl-sn-Glycero -3-Phosphoethanolamine (DSPE) and DSPE-PEG 2000 was obtained from Ponsure Biopharma Co., Ltd (Shanghai, China). Soybean phosphatidylcholine (SPC) and cholesterol (CHOL) were obtained from Sinopharm Chemical Reagent (Shanghai, China). PC-9 and H1975 lung cancer cell line was provided by the Institute of Biochemistry and Cell Biology, Chinese Academy of Sciences (Shanghai, China). All the other reagents used were of analytical grade without further purification.

\section{Preparation of GFT/CUR LPs}

GFT/CUR LPs were prepared by the method of emulsification and solvent evaporation. Briefly, GFT (5 mg), CUR (5 mg), CHOL (10 mg), DSPE-PEG 2000 (15 mg) and SPC (100 mg) were dissolved in $5 \mathrm{~mL}$ chloroform/ methanol $(2: 1, \mathrm{v} / \mathrm{v})$ to produce an oil phase. This was attached to a rotary evaporator to remove the organic phase at $45 \pm 2{ }^{\circ} \mathrm{C}$, leading to the formation of a film on the flask wall. The lipid membranes were hydrated with $5 \mathrm{~mL}$ PBS (pH 7.0) at $37^{\circ} \mathrm{C}$ for $30 \mathrm{~min}$ and disrupted in ultrasonic homogenizer for $10 \mathrm{~min}$ at $20^{\circ} \mathrm{C}$. Single drug or blank liposome was prepared using the same method as the control group. ${ }^{20}$

\section{Physicochemical Characterization}

Size distribution and zeta potential of LPs were measured using the Zetasizer Nano ZS (Malvern Instruments, UK). Size distribution was reported as particle diameter along with polydispersity index (PDI). The encapsulation efficiency (EE) of GFT and CUR in LPs was calculated by the following equation:

$$
\mathrm{EE} \%=\left(\mathrm{W}_{\text {total }}-\mathrm{W}_{\text {free }}\right) / \mathrm{W}_{\text {total }} \times 100 \%
$$

where $\mathrm{EE} \%$ is the entrapment efficiency, $\mathrm{W}_{\text {free }}$ the amount of free drug in the LPs sample, and $\mathrm{W}_{\text {total }}$ the total amount of drug in LPs. Three separate characterizations were conducted for each formulation and results are reported as mean \pm standard deviation (SD).

\section{Stability}

According to the guidelines provided by the International Conference on Harmonisation, experiments on the stability of all LPs were carried out at a temperature of $25^{\circ} \mathrm{C} \pm 2{ }^{\circ} \mathrm{C}$ and a relative humidity of $60 \% \pm 5 \%$ for one to three months. All physicochemical characteristics of the stored samples were determined. 


\section{In vitro Release}

The in vitro drug release was carried out as described below. After different LPs $(10 \mathrm{mg})$ were suspended in dialysis bags (Mol $\approx 8000 \sim 10,000$ ), the bags were placed in a tube and vibrated horizontally $\left(50 \mathrm{rpm}, 37^{\circ} \mathrm{C}\right)$. The release medium included $200 \mathrm{~mL}$ PBS $(\mathrm{pH}=7.4)$ and $10 \%$ human plasma. At designated time intervals $(0.5,1,2,4,8$, $12,16,24,32$ and $48 \mathrm{~h}$ ), $1 \mathrm{~mL}$ of dialysate was taken for analysis and replaced by $1 \mathrm{~mL}$ of fresh medium. The dialysate was removed and centrifuged at $12,000 \mathrm{rpm}$ for 10 min and then analyzed by HPLC.

\section{In vitro Cellular Uptake}

In order to examine the cellular distributions of LPs, coumarin-6 was encapsulated into LPs as a fluorescent marker and the fluorescence was observed through a confocal laser scanning microscopy and quantified by a microplate reader. Briefly, PC-9 or H1975 cells were seeded in 96-well plates under the incubation to reach a density of $1 \times 10^{5} / \mathrm{mL}$. After $24 \mathrm{~h}$, coumarin-6-labeled LPs (equivalent to $0.1 \mu \mathrm{g} / \mathrm{mL}$ of coumarin-6) were added into the medium and after another $2 \mathrm{hrs}$, the cells in well were washed with ice-cold PBS for 3 times to remove the LPs that were not internalized in cells at the predetermined interval. Finally, cells treated with coumarin6-labeled LPs were collected and the internalization of the coumarin-6-labeled LPs was observed using a confocal laser scanning microscopy. The internalization efficiency of LPs in cells was quantified by measuring the fluorescent intensity of internalized coumarin6-labeled LPs.

\section{Cell Viability}

In order to determine the role of all LPs on the cell apoptosis, MTT assay was used to evaluate cell viability. PC-9 or H1975 cells were seeded in 96-well plates $\left(1 \times 10^{5}\right.$ cells $\left./ \mathrm{mL}\right)$ and maintained in Dulbecco's Modified Eagle Medium (DMEM) with $10 \%$ fetal bovine serum (FBS) and $5 \% \mathrm{CO}_{2}$ at $37^{\circ} \mathrm{C}$. The cells were incubated for 48 hours with different concentrations of free GFT, free GFT and free CUR, GFT LPs, GFT LPs combined with CUR LPs, GFT/CUR LPs (GFT concentrations ranging from 0.04 to $10 \mu \mathrm{g} / \mathrm{mL}$ were added, the weight ratio of GFT and CUR was 1:1 when CUR was added). The MTT assay was performed according to the manufacturer's instructions.

\section{In vivo Antitumor}

For antitumor activity, the experiment was performed as described below. Briefly, on day $0,1 \times 10^{6} \mathrm{H} 1975$ cells were injected SC into BALB/c nude mice. After one week, the average tumor volume of each mouse reached about 50 100 $\mathrm{mm}^{3}$. From day 8, mice bearing H1975 xenografts were randomized into 6 groups and treated with LPs or free drugs (blank LPs, free GFT, free GFT and free CUR, GFT LPs, GFT LPs combined with CUR LPs) and GFT/CUR LPs (GFT concentration was $10 \mathrm{mg} /$ $\mathrm{kg}$, the weight ratio of GFT and CUR was 1:1 when CUR was added). Each group contained five mice. All the formulations were given via oral administration. Free GFT and free CUR were dissolved in Tween-80. The drugs were given once every 3 days for 10 times (on days $8,11,14,17,20,23,26,29,32$ and 35). The effect of the drugs on the tumor proportion was evaluated on day 35. The tumor volume was calculated through the equation volume $=$ width $^{2} \times$ length $/ 2$. The weight of each mouse was measured for the evaluation of systemic toxicity. At the end of the study, the mice were sacrificed by $\mathrm{CO} 2$ inhalation, and the tumor tissues were removed and weighed for observation. All animal experiments were performed in accordance with institutional guidelines, following the protocol approved by the Ethics Committees of Shanghai Shidong hospital. National Institutes of Health guide for the care and use of laboratory animals was strictly followed by us.

\section{HPLC Analysis}

The analysis of GFT and CUR levels in vitro was carried out using LC-MS/MS on a system equipped with an Agilent 1100 Series (Agilent Technologies, Santa Clara, CA, USA) and API 2000 (AB, Massachusetts, USA). The column was a Diamonsil C18 HPLC column (Dikma Technologies Inc, Lake Forest, CA, USA; $5 \mu \mathrm{m}, 200 \times 4.6 \mathrm{~mm})$. The mobile phase consisted of $20 \%$ Ammonium acetate $(10 \mathrm{mmol} / \mathrm{L})$ and $80 \%$ acetonitrile, and the flow rate was $0.2 \mathrm{~mL} / \mathrm{min}$. The column temperature was $30^{\circ} \mathrm{C}$.

\section{Statistical Analysis}

Student's $t$-test was used to compare the groups. A $p$ value of less than 0.05 is considered to indicate statistical significance. Chou-Talalay isobologram methods were used to judge the synergy between the two drugs. All data from three independent experiments are expressed as mean \pm standard deviation. 


\section{Results}

\section{Preparation and Characterization}

The LPs were fabricated by emulsification and the solvent evaporation method. The morphology of the prepared LPs was determined by TEM and is shown in Figure 1. The physicochemical properties including particle size, polydispersity index (PDI), zeta potential and encapsulation efficiency (EE) are listed in Table 1. The particle size of GFT LPs was $112.9 \pm 11.9 \mathrm{~nm}$ and slightly increased to $130.5 \pm$ $12.6 \mathrm{~nm}$ after incorporated with CUR. The particle size of CUR LPs was about $200 \mathrm{~nm}$. Zeta-potentials of all LPs ranged from $-13.4 \mathrm{mV}$ to $-22.2 \mathrm{mV}$ and were all slightly negative due to the presence of DSPE-PEG ${ }_{2000}$ on the liposomal surface (DSPE has an anionic head group). EE (\%) of GFT and CUR in all LPs was observed to be above $80 \%$. Interestingly, the co-delivery of GFT and CUR LPs resulted in a slightly lower EE of GFT $(82.4 \pm 3.1 \%)$ as compared to that of GFT LPs $(86.5 \pm 3.6 \%)$. The same was observed in CUR LPs.

\section{Stability}

The stability data of all LPs are summarized in Table 2. In the stability test, LPs maintained a good round shape and no physicochemical characteristics of GFT/CUR LPs showed significant changes. In addition, no aggregation or precipitation of LPs was observed during the 3-month storage. The stability study indicated that a suitable formulation (lyophilized liposomes) increased the storage time of the drug.

\section{In vitro Release}

To examine drug release in vitro, free GFT, GFT LPs and GFT/CUR LPs at the same concentration were loaded in dialysis bags with a molecular weight cutoff of 8000 10,000 and immersed in $200 \mathrm{~mL}$ of PBS. The aliquots from release
Table I The Characteristics of GFT LPs, CUR LPs and GFT/ CUR LPs. $(n=3)$

\begin{tabular}{|c|c|c|c|}
\hline Parameters & GFT LPs & CUR LPs & $\begin{array}{l}\text { GFT/CUR } \\
\text { LPs }\end{array}$ \\
\hline Particle size $(\mathrm{nm})$ & $112.9 \pm 11.9$ & $116.3 \pm 9.6$ & $130.5 \pm 12.6$ \\
\hline Encapsulation & $86.5 \pm 3.6$ & $88.1 \pm 2.6$ & GFT $(82.4 \pm 3.1)$ \\
\hline efficiency (\%) & & & CUR $(81.9 \pm 2.3)$ \\
\hline Polydispersity index & 0.29 & 0.18 & 0.17 \\
\hline Zeta potentials (mV) & $-13.4 \pm 1.5$ & $-15.8 \pm 1.9$ & $-22.2 \pm 1.6$ \\
\hline
\end{tabular}

media were taken out at designated time intervals to determine the release pattern of GFT in media. The same conditions were applied for the determination of CUR (Figure 2). The results showed that compared with the fast release of free drug, drugs encapsulated in LPs, when controlled, could release slowly and smoothly at $\mathrm{pH}$ 7.4. The release curve in PBS could be divided into two phases: an initial fast drug release stage and a stable release stage. The drug was released rapidly in the initial fast release stage but slowly in the latter stable stage through diffusion because of the continuous degradation of the polymer. As shown in Figure 2, throughout the study, a sustained GFT (CUR) release to a total of about $82 \%$ (72\%) was observed in the GFT/CUR LPs group, indicating that GFT/CUR LPs could be used as a lasting and effective drug delivery system.

\section{In vitro Cellular Uptake}

To investigate the cellular uptake of the co-delivery form of GFT/CUR LPs, a fluorescence imaging study was carried out using PC-9 or H1975 cells. Blank LPs were used as a positive control. As shown in Figure 3, in the case of PC-9 cells, cell internalization was higher for GFT/CUR LPs than for others. The results showed that the combination of GFT and CUR

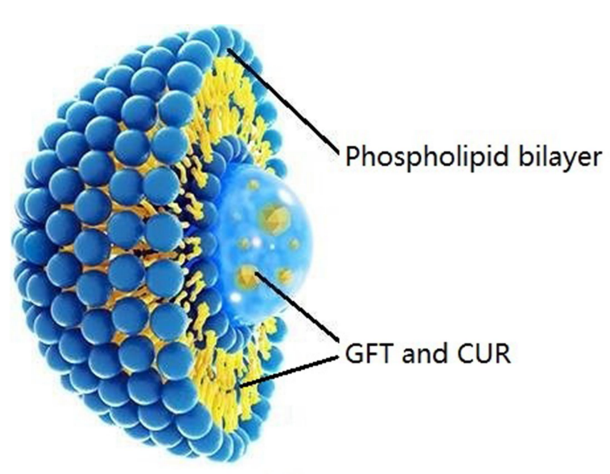

A

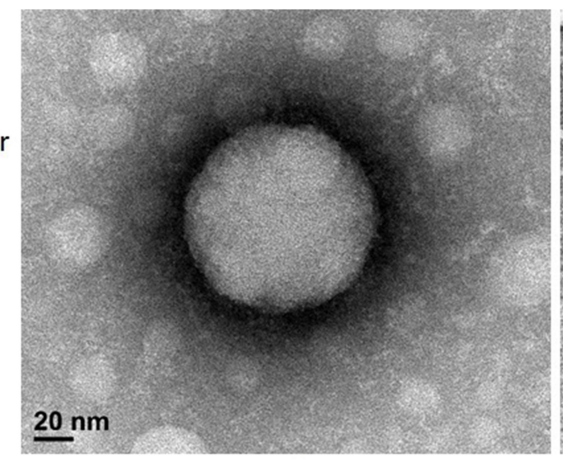

B

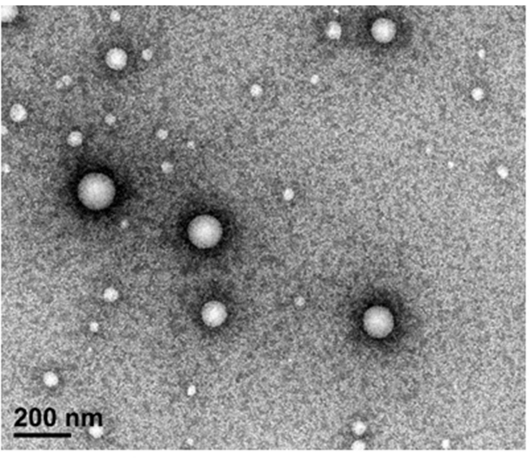

C

Figure I (A) The basic structure of GFT/CUR LPs; (B) The transmission electron microscope of GFT/CUR LPs (Magnification $\times 200,000)$; (C) The transmission electron microscope of GFT/CUR LPs (Magnification ×50,000). 
Table 2 Physical Stability of All LPs at $25^{\circ} \mathrm{C} \pm 2^{\circ} \mathrm{C}$, Relative Humidity $(60 \% \pm 5 \%)(n=3)$

\begin{tabular}{|c|c|c|c|c|c|c|}
\hline & \multicolumn{2}{|l|}{ GFT LPs } & \multicolumn{2}{|l|}{ CUR LPs } & \multicolumn{2}{|l|}{ GFT/CUR LPs } \\
\hline & 0 Day & 3rd Month & 0 Day & 3rd Month & 0 Day & 3rd Month \\
\hline Appearance & Milky white & Milky white & Yellow & Yellow & Creamy yellow & Creamy yellow \\
\hline Particle size $(\mathrm{nm})$ & $112.9 \pm 11.9$ & $116.4 \pm 10.8$ & $116.3 \pm 9.6$ & $118.9 \pm 7.8$ & $130.5 \pm 12.6$ & $|33.4 \pm| \mid .2$ \\
\hline Zeta potential $(\mathrm{mV})$ & $-13.4 \pm 1.5$ & $-15.6 \pm 1.7$ & $-15.8 \pm 1.9$ & $-17.4 \pm 1.6$ & $-22.2 \pm 1.6$ & $-24.6 \pm 2.1$ \\
\hline EE (\%) & $86.5 \pm 3.6$ & $85.1 \pm 2.9$ & $88.1 \pm 2.6$ & $86.6 \pm 3.1$ & GFT $(82.4 \pm 3.1)$ & GFT (8I.6 $6 \pm 2.7)$ \\
\hline & & & & & CUR $(81.9 \pm 2.3)$ & CUR $(80.2 \pm 2.5)$ \\
\hline
\end{tabular}

could enable cells to internalize, allowing more drugs to enter cells successfully. The mean fluorescence intensity (Figure 3B) for GFT/CUR LPs was approximately 1.36 and 1.48 folds higher than those for GFT LPs and CUR LPs, respectively. However, in H1975 cells, the results of cellular uptake were slightly different. The cell internalization of GFT LPs was lower than others and the mean fluorescence intensity was close to that of blank LPs (Figure 3B). The cell internalization of CUR LPs was higher than that of blank LPs. There was obviously synergistic effect in the combination of the two drugs. The mean fluorescence intensity (Figure 3B) for GFT/CUR LPs was approximately 2.69 and 1.43 folds higher than those for GFT LPs and CUR LPs, respectively.

\section{Cell Viability}

To assess the cell viability of all LPs, two cell types were investigated in vitro (PC-9 and H1975). As shown in Figure 4A, with the increase of GFT concentration, the growth of PC-9 cells was significantly inhibited by either free GFT or GFT LPs, and it was dose-dependent. At the same time, it was found that the inhibition effect of GFT LPs was better than that of free GFT. When CUR was added, both free CUR and CUR LPs could increase the inhibitory effect of free GFT or GFT LPs. Among all the preparations, the inhibition effect of GFT/CUR LPs on PC-9 cells was the best, but no statistical difference was observed when it was compared with the data of other

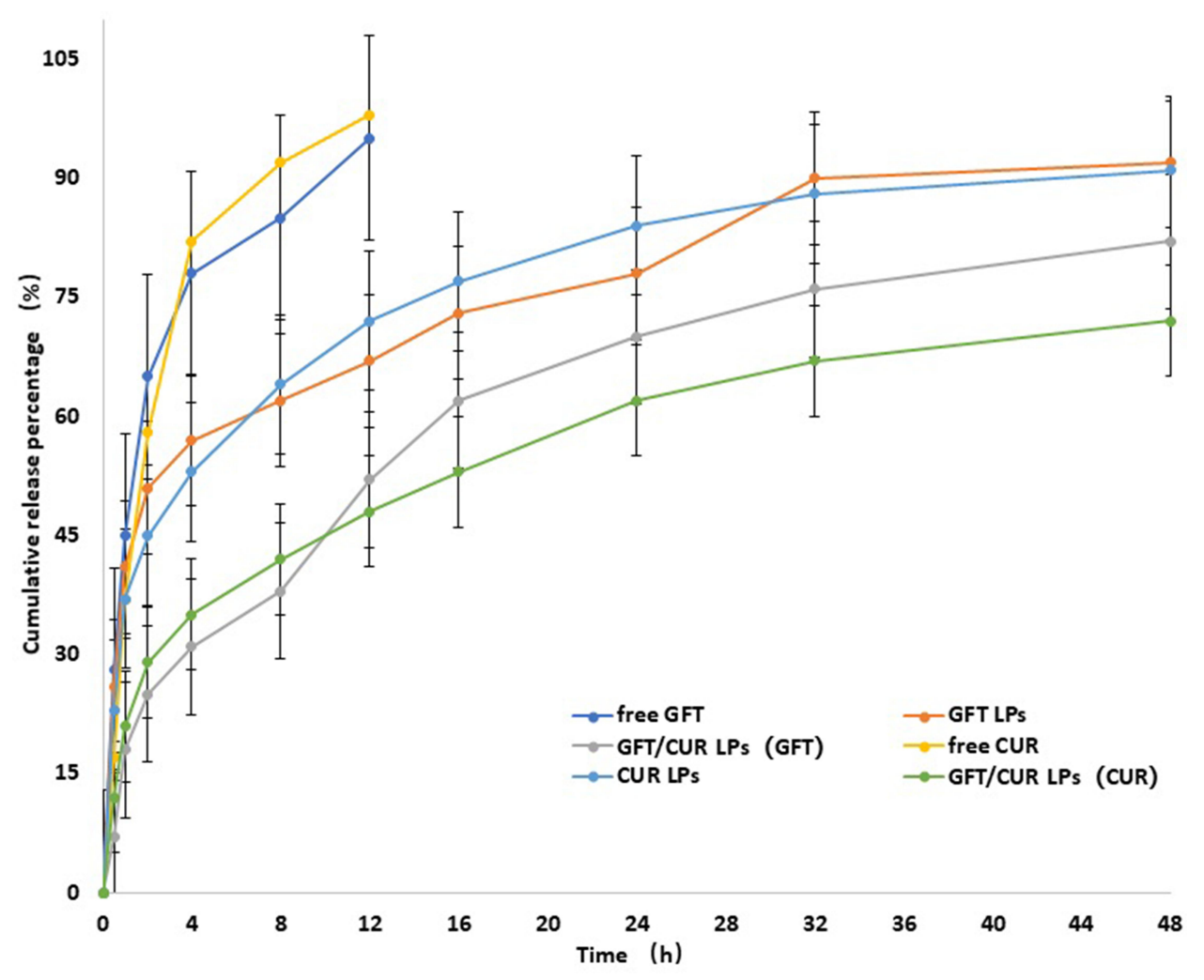

Figure 2 The accumulative release of GFT/CUR LPs and other related in the medium at $\mathrm{pH}=7.4$. The results were expressed as mean \pm SD ( $n=3$ ). 

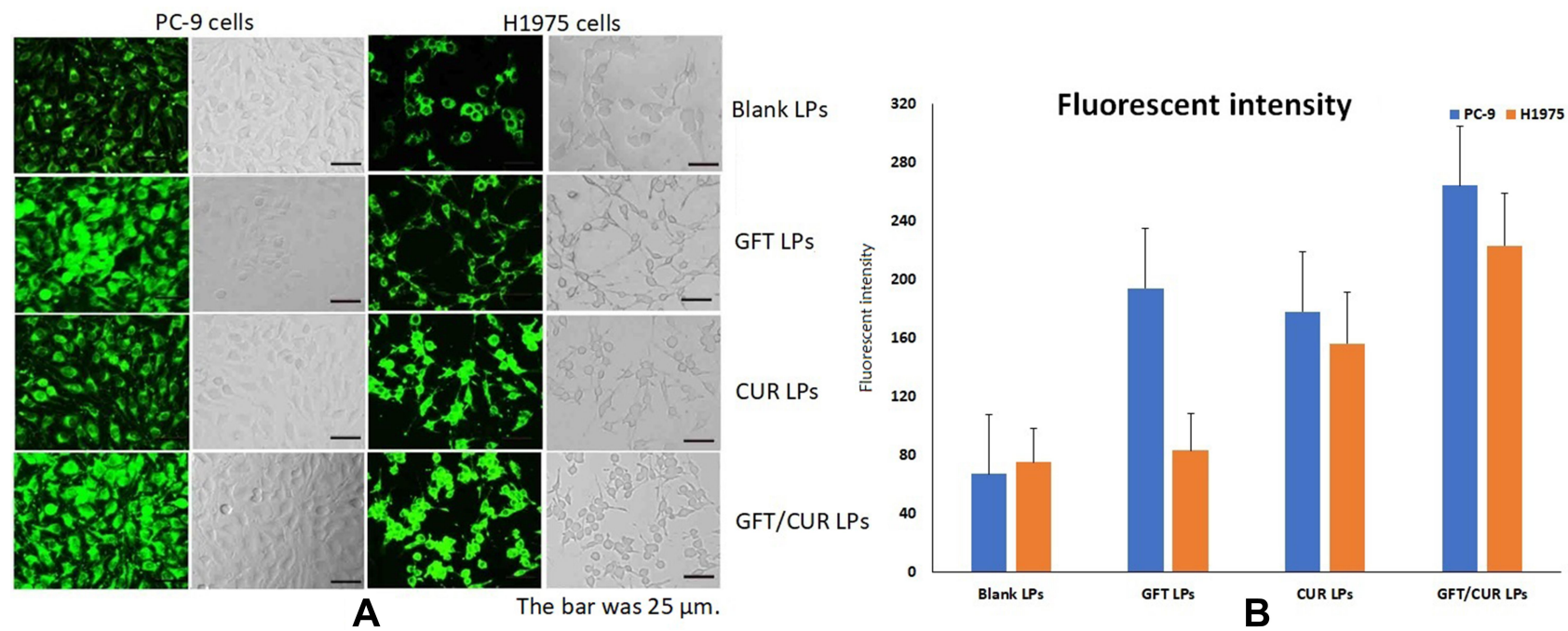

Figure 3 (A) Cellular uptake of coumarin-6-loaded LPs. Blank LPs, GFT LPs, CUR LPs, GFT/CUR LPs for 2 h by PC-9 and HI 975 cells. The bar was $25 \mu$ m. (B) Quantitative results of cellular uptake. (blue) PC-9 cells and (orange) HI975 cells.

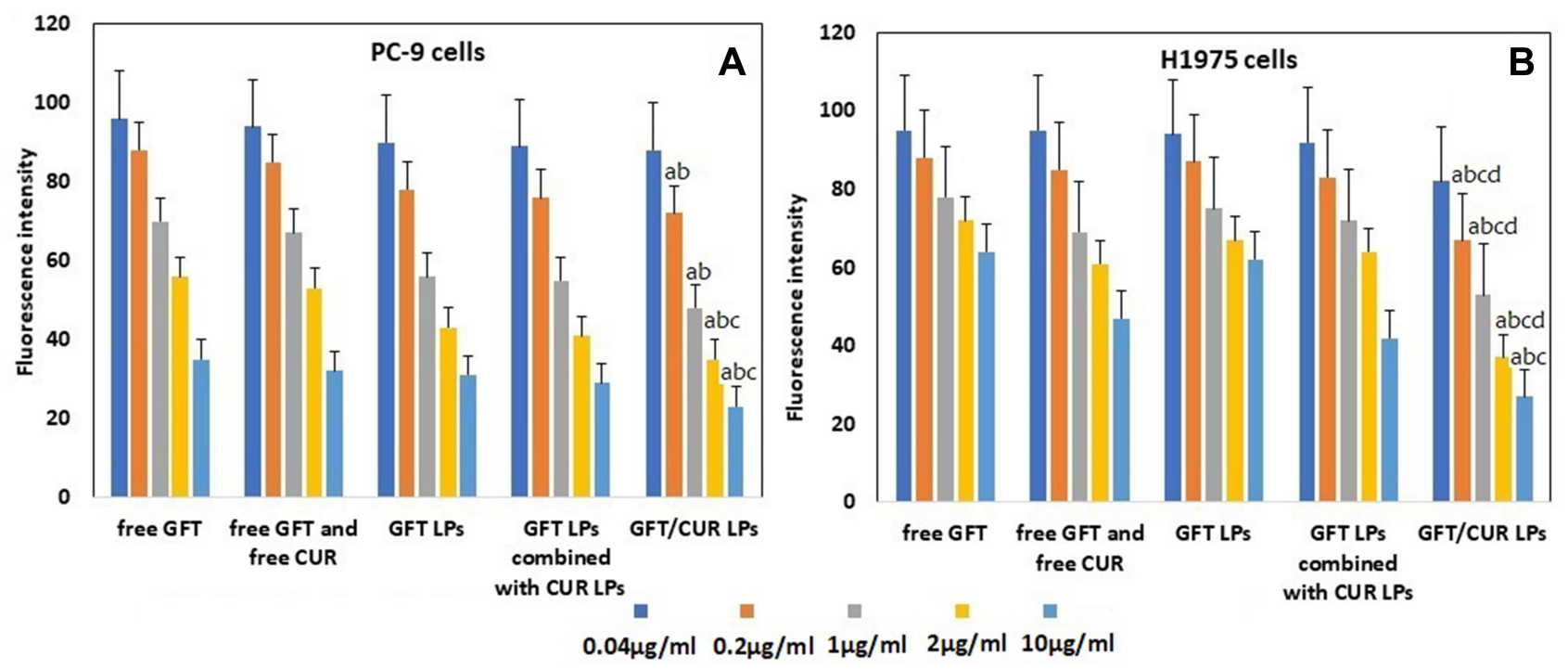

Figure 4 (A) The cellular viability of PC-9 cells cultured with free GFT, free GFT and free CUR, GFT LPs, GFT LPs combined with CUR LPs and GFT/CUR LPs in the incubation time of 24 hours at the five different concentrations $(n=6)$. (B) The cellular viability of HI 975 cells cultured with Blank LPs, free GFT, free CUR, GFT LPs, CUR LPs and GFT/CUR LPs in the incubation time of 24 hours at the five different concentrations $(n=6)$. Data $=$ mean $\pm S D$. ${ }^{a} P<0.05$, compared with free GFT; ${ }^{b}<<0.05$, compared with free GFT and free CUR; ${ }^{c} p<0.05$, compared with GFT LPs; ${ }^{d} p<0.05$, compared with GFT LPs combined with CUR LPs.

groups. As shown in Figure 4B, the inhibitory effects on H1975 cells of both free GFT and GFT LPs were not ideal. When free CUR or CUR LPs were added, a similar inhibitory effect occurred on H1975 cells. In brief, the cytotoxic effect of the co-delivered LPs was comparable or even superior to that of the combination of both free drugs in both cancer cell types, indicating that the LPs of the two drugs did not impair the synergistic antitumor effect in vitro. By using the method of Chou-Talalay isobologram, the results showed that with an increase of concentration, the synergistic effect of compound preparation gradually appeared. When the concentration was higher than $0.2 \mu \mathrm{g} / \mathrm{mL}$, CI index began to be less than 1 , indicating the synergistic effect of GFT/CUR LPs. The CI of GFT/CUR LPs in PC-9 or H1975 cancer cell lines is listed in Table 3.

\section{In vivo Antitumor}

The therapeutic efficacy of all LPs or free drugs was evaluated in nude mice bearing H1975 xenografts 
Table $3 \mathrm{Cl}$ of GFT/CUR LPs in PC-9 or HI975 Cancer Cell Lines. $(n=3)$

\begin{tabular}{|l|l|l|l|l|l|}
\hline GFT Concentration $(\boldsymbol{\mu g} / \mathbf{m L})$ & $\mathbf{0 . 0 4}$ & $\mathbf{0 . 2}$ & $\mathbf{l}$ & $\mathbf{2}$ & 10 \\
\hline $\mathrm{Cl}(\mathrm{PC}-9)$ & $1.23 * 10^{3} \pm 113.2$ & $0.214 \pm 0.021$ & $0.053 \pm 0.008$ & $0.023 \pm 0.004$ & $0.013 \pm 0.003$ \\
$\mathrm{Cl}(\mathrm{HI} 975)$ & $1.15^{*} 10^{3} \pm 107.1$ & $0.116 \pm 0.017$ & $0.068 \pm 0.007$ & $0.025 \pm 0.006$ & $0.015 \pm 0.002$ \\
\hline
\end{tabular}

(Figure 5). After a week of culture, the transplanted tumor could basically take shape. Strikingly, at the end point (day 35), the GFT/CUR LPs treatment achieved the best therapeutic efficacy, resulting in a 3 -fold increase in tumor volume; while free GFT and GFT LPs achieved a 7.6-fold and a 6.0-fold increases in tumor volume, respectively. Free GFT and free CUR, as well as GFT LPs combined with CUR LPs achieved moderate therapeutic efficacy, resulting in a 5.5-fold and a 4.5 -fold increases in tumor volume, respectively. The toxicity of all treatments was examined by monitoring the body weight (Figure 5D). The body weights of the mice in all groups gradually increased, suggesting that the mice could tolerate all the treatments well.

\section{Discussion}

In this study, we prepared GFT/CUR LPs with excellent properties representing good drug entrapment, sustained release, small particle size, low polydispersity index, and high encapsulation efficiency. Theoretically, both drugs
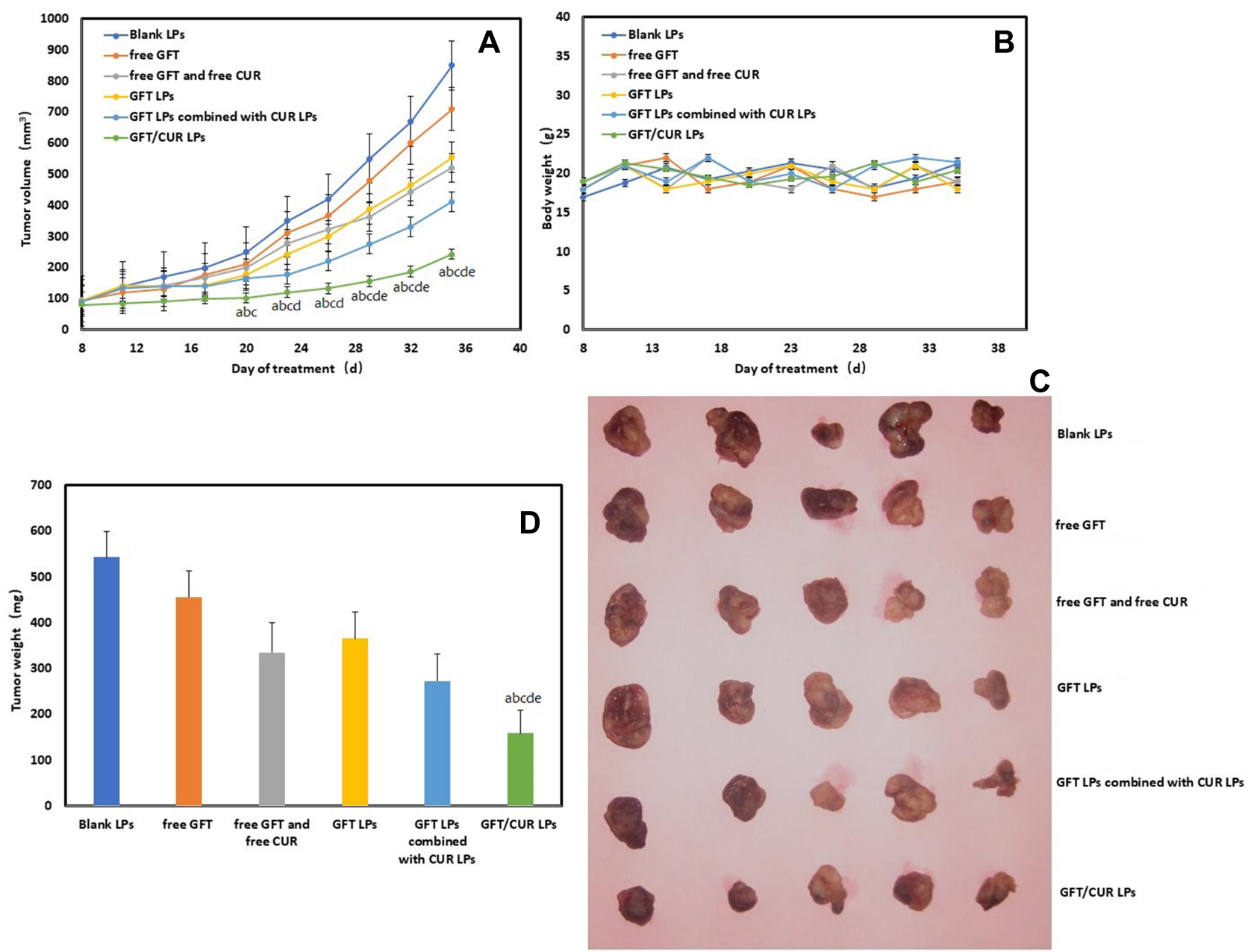

Figure 5 In vivo antitumor of all GFT formulations HI975 tumor-bearing mouse model. (A) The tumor volume (mm ${ }^{3}$ ) change is presented as a function of time after drug treatment. (B) Body weight profiles of mice upon treatment. (C) Image showing tumors excised from the mice. (D) The tumor tissue weight on day 35 , the endpoint of the study. ${ }^{a} \mathrm{P}<0.05$, compared with blank LPs; ${ }^{b} \mathrm{p}<0.05$, compared with free GFT; ${ }^{\mathrm{c}} \mathrm{p}<0.05$, compared with free GFT and free CUR; ${ }^{\mathrm{d}} \mathrm{P}<0.05$, compared with GFT LPs; ${ }^{\mathrm{e}} \mathrm{p}<$ 0.05 , compared with GFT LPs combined with CUR LPs. 
could show synergistic effect because GFT and CUR have different cytotoxic mechanisms. It is known that GFT induces the apoptosis of cancer cells by inhibiting EGFR tyrosine kinase. $^{21}$ On the other hand, CUR is known to block the nuclear factor $(\mathrm{NF})-\kappa \mathrm{B}$, a transcriptional factor that is a major inhibitor of cell proliferation, apoptosis and resistance. $^{18,19}$ Hence, a co-treatment of GFT and CUR could better enhance cytotoxicity against cancer cells compared with the conventional single chemotherapy. The particle size range of all LPs was shown to be near 200 $\mathrm{nm}$. Such physical characteristic of all LPs in the range of $10-200 \mathrm{~nm}$ is likely to give LPs advantages in exploiting the enhanced permeation and retention (EPR) effect against solid tumors through the leaky vasculature. ${ }^{22-25}$ According to the results of in vitro release, drug released during the first $48 \mathrm{~h}$ accounted for over $80 \%$ of the total volume and the remnants were released over a longer period of incubation. The main reason for this was the electrostatic repulsion between molecules of DSPE$\mathrm{PEG}_{2000}$. The molecular chains of DSPE-PEG 2000 tended to extend and were easily degraded into small parts, thus facilitating the diffusion of drugs into the media.

Our combination therapy achieved superior therapeutic efficacy, as reflected by the fact that the mean tumor volume was significantly lowered and no significant body weight loss was observed compared with other groups. The mechanism underlying the superior antitumor activity of the combination therapy could be explained from two perspectives. In the first step, GFT/CUR LPs could efficiently accumulate in tumors due to their long circulation and the EPR effect. Secondly, incorporating two drugs (GFT and CUR) into one LPs carrier boosted the synergistic effect.

From the results of cell uptake and cell viability, it was also the first time to attempt to verify whether the combination of GFT and CUR could produce a synergistic effect against cancer cells, especially GFT-resistant H1975 cells, when formulated into LPs and co-delivered. Thirdly, there is literature reporting that GFT is the substrate of ABCG2/ BCRP, a member of the ABC (ATP binding cassette transporter) protein family, and that CUR is an effective inhibitor of ABCG2 expression level. ${ }^{26-28}$ Moreover, CUR could enhance the concentration of GFT in PC-9 or H1975 cell membrane to a certain extent. This is especially important for H1975 cells due to its GFT-resistant properties.

\section{Conclusion}

In this study, we prepared LPs which can embed the anticancer drug GFT and CUR, and investigated whether they could enhance the antitumor effects of anticancer drugs against MDR. The LPs system was characterized by TEM, particle size, encapsulation efficiency, cellular uptake and cell viability. In addition, the release characteristics and pharmacodynamics of the LPs were also studied in detail. The results showed that GFT/CUR LPs were characterized by a small particle size of about $130 \mathrm{~nm}$ and a negative zeta potential of about $-22.2 \mathrm{mV}$, and that the drug was controlled to release slowly on a biphasic pattern. Compared with the control groups, GFT/CUR LPs showed a higher cellular uptake and cell inhibition rate. Through pharmacodynamics analysis, we found that incorporating two drugs (GFT and CUR) into one LPs carrier could boost the synergistic effect. The co-delivery of GFT and CUR has the potential to improve cancer treatment efficacy and overcome acquired resistance, especially towards GFT-resistant cells.

\section{Disclosure}

The authors report no conflicts of interest for this work.

\section{References}

1. Osmani L, Askin F, Gabrielson E, Li QK. Current WHO guidelines and the critical role of immunohistochemical markers in the subclassification of non-small cell lung carcinoma (NSCLC): moving from targeted therapy to immunotherapy. Semin Cancer Biol. 2018;52(Pt 1):103-109. doi:10.1016/j.semcancer.2017.11.019

2. Jonna S, Subramaniam DS. Molecular diagnostics and targeted therapies in non-small cell lung cancer (NSCLC): an update. Discov Med. 2019;27(148):167-170.

3. Recondo G, Facchinetti F, Olaussen KA, Besse B, Friboulet L. Making the first move in EGFR-driven or ALK-driven NSCLC: first-generation or next-generation TKI? Nat Rev Clin Oncol. 2018;15(11):694-708. doi:10.1038/s41571-018-0081-4

4. Yang Z, Tam KY. Combination Strategies Using EGFR-TKi in NSCLC Therapy: learning from the Gap between Pre-Clinical Results and Clinical Outcomes. Int J Biol Sci. 2018;14(2):204-216.

5. Rawluk J, Waller CF. Gefitinib. Recent Results Cancer Res. 2018; 211:235-246.

6. Budha NR, Frymoyer A, Smelick GS, et al. Drug absorption interactions between oral targeted anticancer agents and PPIs: is pH dependent solubility the Achilles heel of targeted therapy? Clin Pharmacol Ther. 2012;92(2):203-213.

7. EGFR Tyrosine Kinase Inhibitor, 2016. Product monograph IRESSA $^{\circledR}$. Epidermal Growth Factor Receptor (EGFR) Tyrosine Kinase Inhibitor. Available from: https://www.drugs.com/cdi/gefiti nib.html. Accessed September 4, 2016.

8. Wu SG, Shih JY. Management of acquired resistance to EGFR TKI-targeted therapy in advanced non-small cell lung cancer. Mol Cancer. 2018;17(1):38.

9. Liu Z, Gao W. Overcoming acquired resistance of gefitinib in lung cancer cells without T790M by AZD9291 or Twist1 knockdown in vitro and in vivo. Arch Toxicol. 2019;93(6):1555-1571.

10. Gao J, Li H-R, Jin C, Jiang J-H, Ding J-Y. Strategies to overcome acquired resistance to EGFR TKI in the treatment of non-small cell lung cancer. Clin Transl Oncol. 2019;21(10):1287-1301. doi:10.10 07/s12094-019-02075-1 
11. Wan Y, Yuan Y, Pan Y, Zhang Y. Antitumor activity of high-dose pulsatile gefitinib in non-small-cell lung cancer with acquired resistance to epidermal growth factor receptor tyrosine kinase inhibitors. Exp Ther Med. 2017;13(6):3067-3074. doi:10.3892/etm.2017.4356

12. Singla AK, Garg A, Aggarwal D. Paclitaxel and its formulations. Int J Pharm. 2002;2(35):179-192.

13. Baliga MS, Joseph N, Venkataranganna MV, Saxena A, Ponemone V, Fayad R. Curcumin, an active component of turmeric in the prevention and treatment of ulcerative colitis: preclinical and clinical observations. Food Funct. 2012;3(11):1109-1117. doi:10.1039/c2fo 30097d

14. Yallapu MM, Jaggi M, Chauhan SC. Curcumin nanoformulations: a future nanomedicine for cancer. Drug Discov Today. 2012;17(1-2):71-80. doi:10.1016/j.drudis.2011.09.009

15. Vyas A, Dandawate P, Padhye S, Ahmad A, Sarkar F. Perspectives on new synthetic curcumin analogs and their potential anticancer properties. Curr Pharm Des. 2013;19(11):2047-2069.

16. Anand P, Nair HB, Sung B, et al. RETRACTED: design of curcumin-loaded PLGA nanoparticles formulation with enhanced cellular uptake, and increased bioactivity in vitro and superior bioavailability in vivo. Biochem Pharmacol. 2010;79(3):330-338. doi:10. 1016/j.bcp.2009.09.003

17. Nair KL, Thulasidasan AKT, Deepa G, Anto RJ, Kumar GS. Purely aqueous PLGA nanoparticulate formulations of curcumin exhibit enhanced anticancer activity with dependence on the combination of the carrier. Int $J$ Pharm. 2012;425(1-2):44-52. doi:10.1016/j. ijpharm.2012.01.003

18. Choi BH, Kim CG, Lim Y, Shin SY, Lee YH. Curcumin downregulates the multidrug-resistance mdrlb gene by inhibiting the PI3K/Akt/NFкB pathway. Cancer Lett. 2008;259(1):111-118. doi:10. 1016/j.canlet.2007.10.003

19. Neerati P, Sudhakar YA, Kanwar JR. Curcumin Regulates Colon Cancer by Inhibiting P-Glycoprotein in In-situ Cancerous Colon Perfusion Rat Model. J Cancer Sci Ther. 2013;5:313-319.
20. Wang XB, Guan QG, Chen W, Hu XM, Li L. Novel nanoliposomal delivery system for polydatin: preparation, characterization, and in vivo evaluation. Drug Des Dev Ther. 2015;9:1805-1813.

21. Zhao C, Han SY, Li PP. Pharmacokinetics of Gefitinib: roles of Drug Metabolizing Enzymes and Transporters. Curr Drug Deliv. 2017;14 (2):282-288.

22. Kalyane D, Raval N, Maheshwari R, Tambe V, Kalia K, Tekade RK. Employment of enhanced permeability and retention effect (EPR): nanoparticle-based precision tools for targeting of therapeutic and diagnostic agent in cancer. Mater Sci Eng C Mater Biol Appl. 2019;98:1252-1276. doi:10.1016/j.msec.2019.01.066

23. Stylianopoulos T. EPR-effect: utilizing size-dependent nanoparticle delivery to solid tumors. Ther Deliv. 2013;4(4):421-423. doi:10.41 55/tde. 13.8

24. Kesharwani SS, Ahmad R, Bakkari MA, et al. Site-directed non-covalent polymer-drug complexes for inflammatory bowel disease (IBD): formulation development, characterization and pharmacological evaluation[J]. J Control Release. 2018;290:165-179. doi:10.1016/j.jconrel.2018.08.004

25. Kumar S, Kesharwani SS, Mathur H, et al. Molecular complexation of curcumin with $\mathrm{pH}$ sensitive cationic copolymer enhances the aqueous solubility, stability and bioavailability of curcumin. Eur J Pharm Sci. 2016;82:86-96. doi:10.1016/j.ejps.2015.11.010

26. Kusuhara H, Furuie H, Inano A, et al. Pharmacokinetic interaction study of sulphasalazine in healthy subjects and the impact of CURcumin as an in vivo inhibitor of BCRP[J]. Br J Pharmacol. 2012;166(6):1793-1803. doi:10.1111/j.1476-5381.2012.01887.x

27. Shukla S, Zaher H, Hartz A, et al. Curcumin inhibits the activity of ABCG2/BCRP1, a multidrug resistance-linked ABC drug transporter in mice[J]. Pharm Res. 2009;26(2):480-487. doi:10.1007/s11095008-9735-8

28. Chen P, Huang HP, Wang Y, et al. Curcumin overcome primary gefitinib resistance in non-small-cell lung cancer cells through inducing autophagy-related cell death[J]. J Exp Clin Cancer Res. 2019;38 (1):254.

\section{Publish your work in this journal}

OncoTargets and Therapy is an international, peer-reviewed, open access journal focusing on the pathological basis of all cancers, potential targets for therapy and treatment protocols employed to improve the management of cancer patients. The journal also focuses on the impact of management programs and new therapeutic agents and protocols on patient perspectives such as quality of life, adherence and satisfaction. The manuscript management system is completely online and includes a very quick and fair peer-review system, which is all easy to use. Visit http://www.dovepress.com/ testimonials.php to read real quotes from published authors. 